\title{
Microscopic theory of single-electron tunneling through molecular-assembled metallic nanoparticles
}

\author{
Yongqiang Xue * and Mark A. Ratner \\ Department of Chemistry and Materials Research Center, Northwestern University, Evanston, IL 60208
}

(Dated: November 4, 2018)

\begin{abstract}
We present a microscopic theory of single-electron tunneling through metallic nanoparticles connected to the electrodes through molecular bridges. It combines the theory of electron transport through molecular junctions with the description of the charging dynamics on the nanoparticles. We apply the theory to study single-electron tunneling through a gold nanoparticle connected to the gold electrodes through two representative benzene-based molecules. We calculate the background charge on the nanoparticle induced by the charge transfer between the nanoparticle and linker molecules, the capacitance and resistance of molecular junction using a first-principles based Non-Equilibrium Green's Function theory. We demonstrate the variety of transport characteristics that can be achieved through "engineering" of the metal-molecule interaction.
\end{abstract}

Chemically tailored metallic and semiconducting nanoparticles and their assemblies have become the model system for studying the fundamental physics and chemistry of nanostructured materials $\stackrel{\underline{\underline{1}}}{\underline{\underline{T}}}$ The advancement of the fabrication process using self-assembly $\underline{\underline{\underline{1}}}$ or biodirected-assembly technique ${ }^{2}$ with molecular recognition has made devices based on single and assembled nanoparticles attractive candidates in applications including single-electronics, novel biosensors and nanophotonic devices ${ }^{1,2,3.4}$ For single-electron device applications, the possibility of accurate control over particle size and density provides a substantial advantage over conventional granular metal thin films and suggests possible room-temperature operation $\stackrel{1.3}{=}$ Understanding electrical transport in such metal-molecule composite systems is therefore an important problem.

In the "orthodox" theory of single-electron tunneling through a metallic island separated from the electrodes by insulating gap,,$\frac{5}{5}$ the physics of Coulomb blockade can be well understood using simple circuit-level theory, with circuit parameters including junction resistance, capacitance and charging energy often obtained by fitting experimental data and/or using simple electrostatic considerations. In contrast, for the nanoparticle-based single-electron devices, the transfer of single electron is achieved by tunneling through the linker molecule connecting the nanoparticles to the electrodes and to each other $\stackrel{3.4}{~ T h e ~ l i n k e r ~ m o l e c u l e s ~ c a n ~ b e ~ c o u p l e d ~ s t r o n g l y ~ t o ~ t h e ~ e l e c t r o d e s ~ a n d ~ n a n o p a r t i c l e s ~}$ through appropriate end groups, and the tunneling barrier is induced by the energy mismatch of the molecular levels relative to the metal Fermi-level $\underline{\underline{6}}$ For the molecular junction, the metallic screening of the applied electric field occurs over a distance comparable to the size of the molecule $e^{7.8}$. Consequently, the capacitance of the molecular junction becomes an electro-chemical quantity rather than a geometrical quantity, whose evaluation requires a selfconsistent analysis of the charge/potential response within the molecular junctions ${ }^{9}$ In addition, charge transfer

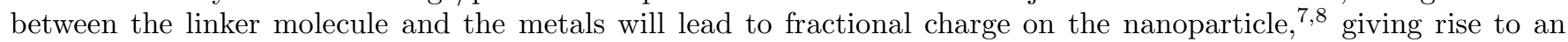
intrinsic background charge (even in the absence of charged impurities and gate voltages), which varies in general with the applied bias voltage and may affect significantly the current-voltage characteristics $\underline{\underline{10}}$ Better understanding of such molecular-assembled single-electron devices therefore requires combining the single-electron tunneling effect with a microscopic description of the electronic processes in the molecular tunnel junctions.

In this paper we describe a microscopic theory of single-electron tunneling through molecular-assembled metallic nanoparticles by combining a first-principles Non-Equilibrium Green's Function (NEGF) theory of molecular transport ${ }^{7.8}$ with a real-time perturbation theory of the reduced density matrix describing the charging dynamics of the metallic nanoparticles $\stackrel{11.12}{1}$ In the lowest-order perturbation theory in the reduced conductance of the molecular junction $\alpha_{0}=\frac{h}{2 e^{2} R_{T}}\left(R_{T}\right.$ is the junction resistance), the theory formally reduces to the rate equation of the "orthodox" theory of Coulomb blockade,,$\frac{5}{5}$ but allows us to take full account of metal-molecule interaction at the atomic scale. In particular, the background charge, capacitance and tunneling rate can all be obtained from the first-principles theory of the molecular tunnel junction. We apply the theory to single-electron tunneling through gold nanoparticles connected to the gold electrodes through two benzene-based molecules-biphenyl dithiolate (BPD) and difluorobenzene (FBF) molecules and show the the variety of current/conductance-voltage characteristics that can obtained through "engineering" of the metal-molecule interaction.

A schematic illustration of the double-barrier tunneling junction is shown in Fig. 11 The system is described by the following Hamiltonian:

$$
H=H_{L}+H_{R}+H_{I}+V+H_{M_{1}}+H_{M_{2}}+H_{T_{1}}+H_{T_{2}} .
$$

where $H_{\alpha}=\sum_{k} \epsilon_{k \alpha} a_{k \alpha}^{\dagger} a_{k \alpha}$ and $H_{I}=\sum_{l} \epsilon_{l I} c_{l I}^{\dagger} c_{l I}$ describe the noninteracting electrons in the two leads $(\alpha=L, R)$ and on the metallic nanoparticle (I) respectively. The indices $k$ and $l$ enumerate the electron states of the leads 
and the nanoparticle. The Coulomb repulsion on the nanoparticle $V$ is obtained from electrostatic considerations as $V(\hat{N})=E_{C}\left(\hat{N}-n_{x}\right)^{2}$, where $E_{C}$ is the charging energy. The background charge $e n_{x}$ can be separated into an intrinsic background charge $e n_{x 0}$ existing at zero voltage and voltage-dependent polarization charge $e n_{x}=Q_{P}\left(V_{L}, V_{R}\right)+e n_{x 0}$. The junction capacitances are obtained from $C_{\alpha}=\frac{\partial Q_{P}}{\partial V_{\alpha}}$, where $V_{\alpha}, C_{\alpha}(\alpha=L, R)$ are the voltage and capacitance of the two tunnel junctions respectively. The charging energy is $E_{C}=e^{2} / 2 C_{\Sigma}$ and the total capacitance $C_{\Sigma}=$ $C_{L}+C_{R}+C_{S}$ is the summation of the two junction capacitances and the self-capacitance $C_{S}$ of the nanoparticle. Here the background charge $e n_{x}$ is induced by the charge transfer between the nanoparticle and the linker molecules, whose value at zero bias voltage gives the intrinsic background charge $e n_{x 0}$.

We assume the molecules can be described by an effective single-particle Hamiltonian $H_{M_{i}}=\sum_{m} \epsilon_{i m} b_{i m}^{\dagger} b_{i m}, i=1,2$. The transfer of single electrons is mediated by tunneling through the molecular bridges $H_{T_{i}}=\sum_{m k l}\left(t_{m k \alpha} b_{i m}^{\dagger} a_{k \alpha}+\right.$ $t_{m l I} b_{i m}^{\dagger} c_{l I} e^{-i \hat{\phi}}+$ C.C.), where $\alpha$ denotes L (R) for molecule 1 (2). The operators $e^{ \pm i \hat{\phi}}$ keep track of the change of the charge on the metallic nanoparticle by $\pm e^{5.11 .13}$ The phase operator $\hat{\phi}$ is the quantum-mechanical conjugate of the excess electron number $\hat{N}$ on the nanoparticle. The charging state $\hat{N}$ is treated independently of the degrees of freedom described by the fermionic field operators $c_{l I}^{\dagger}, c_{l I}$, which is a good approximation for metallic nanoparticles. 5.11 The two electrodes as well as the nanoparticle are treated as large equilibrium reservoirs with corresponding Fermi distribution $f_{i}(E)=f\left(E-\mu_{i}\right), i=L, R, I$. Note that the partition of the voltage drop between the two molecular junctions is determined by the capacitance ratio $V_{L} / V_{R}=\left(\mu_{I}-\mu_{L}\right) /\left(\mu_{R}-\mu_{I}\right)=C_{R} / C_{L}$ and $e V=\mu_{R}-\mu_{L}$.

Following the standard procedure in NEGF theory of mesoscopic transport, 14 the current flowing through the left contact is given by

$$
\begin{aligned}
I_{L}(t) & =\frac{e d \hat{N}_{L}}{d t}=\frac{2 e}{h} \operatorname{Re}\left[\sum_{m k L} t_{m k L} G_{k L m}^{<}(t, t)\right] \\
& =\frac{2 e}{h} \int d t^{\prime} \operatorname{Tr}\left[G_{M_{1}}^{>}\left(t, t^{\prime}\right) \Sigma_{L}^{<}\left(t^{\prime}, t\right)-G_{M_{1}}^{<}\left(t, t^{\prime}\right) \Sigma_{L}^{>}(t, t)\right]
\end{aligned}
$$

where the Green's functions are defined in the standard manner ${ }^{14}$ The self-energy operator $\Sigma_{L}^{<(>)}$describes the interaction with the left electrode $\Sigma_{L ; m n}^{<(>)}\left(t, t^{\prime}\right)=\sum_{k} t_{m k l}(t) g_{L ; k}^{<(>)}\left(t, t^{\prime}\right) t_{n k L}^{*}\left(t^{\prime}\right)\left(g_{L}^{<(>)}\right.$is the corresponding Green's function of the bare left electrode). Similar definition applies to the self-energy operators of the right electrode (R) and the central island (I). Deriving the equation of motion of the Green's function and decoupling the charging dynamics from the single-electron dynamics on the metallic nanoparticle $\left\langle c_{l I}^{\dagger}(t) c_{l I}\left(t^{\prime}\right) e^{i \hat{\phi}\left(t^{\prime}\right)} e^{-i \hat{\phi}(t)}\right\rangle=\left\langle c_{l I}^{\dagger}(t) c_{l I}\left(t^{\prime}\right)\right\rangle\left\langle e^{i \hat{\phi}\left(t^{\prime}\right)} e^{-i \hat{\phi}(t)}\right\rangle$, we obtain the current as 15

$$
\begin{aligned}
I_{L}=- & 2 e i \int \frac{d E}{2 \pi h} \tilde{T}_{L}(E, V) \int d w \\
& {\left[f_{L}(E)\left(1-f_{I}(E-w)\right) C^{>}(w)+\left(1-f_{L}(E)\right) f_{I}(E-w) C^{<}(w)\right], }
\end{aligned}
$$

where $C^{<(>)}(w)$ are the Fourier transform of the correlation functions $C^{<}\left(t, t^{\prime}\right)=i\left\langle e^{i \hat{\phi}\left(t^{\prime}\right)} e^{-i \hat{\phi}(t)}\right\rangle$, $C^{>}\left(t, t^{\prime}\right)=-i\left\langle e^{-i \hat{\phi}(t)} e^{i \hat{\phi}\left(t^{\prime}\right)}\right\rangle$. The "renormalized" transmission function is obtained from $\tilde{T}_{L}(E, V)=$ $\operatorname{Tr}\left[\Gamma_{L}(E, V) \tilde{G}_{M_{1}}^{r}(E, V) \Gamma_{I}(E, V) \tilde{G}_{M_{1}}^{a}(E, V)\right]$ and the "renormalized" Green's function is $G_{M 1}^{r(a)}(E, V)=\left(E \pm 0^{+}-\right.$ $\left.H_{M 1}-\Sigma_{L}^{r(a)}(E, V)-\int d w \Sigma_{I}^{r(a)}(E-w, V) C^{r(a)}(w)\right)^{-1}$ where $C^{r(a)}(w)$ are the Fourier transform of $C^{r(a)}\left(t, t^{\prime}\right)=$ $\pm \theta\left( \pm\left(t-t^{\prime}\right)\right)\left(C^{>}\left(t, t^{\prime}\right)-C^{<}\left(t, t^{\prime}\right)\right)$.

Eq. (3) can be recast into a form suitable for perturbation expansion

$$
\begin{aligned}
I_{L} & =-i e \int d w\left[\alpha_{L}^{+}(w) C^{>}(w)+\alpha_{L}^{-}(w) C^{<}(w)\right], \\
\alpha_{L}^{+}(w) & =\frac{2}{2 \pi h} \int d E \tilde{T}_{L}(E, V) f_{L}(E)\left(1-f_{I}(E-w)\right), \\
\alpha_{L}^{-}(w) & =\frac{2}{2 \pi h} \int d E \tilde{T}_{L}(E, V)\left(1-f_{L}(E)\right) f_{I}(E-w),
\end{aligned}
$$

where $\alpha_{L}^{+(-)}$is the rate for electron tunneling into (out of) the nanoparticle through the left molecular bridge. A similar equation can be written down for the current flowing through the right contact $I_{R}=-i e \int d w\left[\alpha_{R}^{+}(w) C^{>}(w)+\right.$ $\left.\alpha_{R}^{-}(w) C^{<}(w)\right]$ where the tunneling rate $\alpha_{R}^{+(-)}$is evaluated from the transmission coefficient through the right molecular junction and the corresponding Fermi distributions. The condition for current conservation $I_{L}+I_{R}=0$ leads to

$$
\int d w\left[\alpha^{+}(w) C^{>}(w)+\alpha^{-}(w) C^{<}(w)\right]=0,
$$


where $\alpha^{+(-)}(w)=\alpha_{L}^{+(-)}(w)+\alpha_{R}^{+(-)}(w)$. Eqs. (4) and (5) are the central result of this paper which separate the description of electron tunneling through the molecular bridges from that of the charging dynamics of the nanoparticle through the correlation functions $C^{<(>)}$.

The theory of the correlation functions $C^{<(>)}$has been developed by Schöller,Schön and coworkers in a series of publications based on a real-time perturbation theory of the reduced density matrix describing the charging state $\hat{N}$ of the metal island, $\frac{11.12}{12}$ where the perturbation parameter is dimensionless conductance of the tunnel junction $\alpha_{0}=h / 2 e^{2} R_{T}$. In particular, the correlation function $C^{<(>)}$can be expressed as the superposition of contributions due to the charging state transition from $n$ to $n+1$ as $C^{<(>)}(w)=\sum_{n} C^{<(>)}(w, n)$. The current conservation condition (5) can also be shown to hold for each part $C^{<(>)}(w, n) \stackrel{12}{=} \int d w\left[\alpha^{+}(w) C^{>}(w, n)+\alpha^{-}(w) C^{<}(w, n)\right]=0$. In the lowest order perturbation theory with respect to $\alpha_{0}, C^{<(>)}(w, n)$ are related to the probability $P_{n}$ of finding $n$ excess electrons as ${ }^{12} C^{<}(w, n)=2 \pi i P_{n+1} \delta\left(w-\Delta_{n}\right)$ and $C^{>}(w, n)=-2 \pi i P_{n} \delta\left(w-\Delta_{n}\right)$. Here $\Delta_{n}$ is the energy difference of the adjacent charging states $\Delta_{n}=V(n+1)-V(n)=E_{C}\left[1+2\left(n-n_{x}\right)\right]$. Substituting the above formulas into Eqs. (4) and (5), we obtain a set of rate equations formally equivalent to the "orthodox" theory of single-electron tunneling

$$
\alpha^{+}\left(\Delta_{n}\right) P_{n}-\alpha^{-}\left(\Delta_{n}\right) P_{n+1}=0,
$$

Compared to the "orthodox" theory, the above theory allows us to take full account of the microscopic description of the electronic processes in the molecular junction. In particular, considerable simplification can be achieved in the "wide-band limit" of the electrodes, i.e., if the surface density of states of the electrodes is approximately constant in the energy interval of $\Delta_{n}$. The "renormalization" of the nanoparticle self-energy and transmission coefficient can then be neglected, which is true for common simple and noble metals and for nanoparticle size sufficiently large that energy quantization can be neglected ${ }^{16}$

Here we apply the theory to single-electron tunneling through a gold nanoparticle connected to two gold electrodes using biphenyl dithiolate (BPD) and difluorobenzene (FBF) molecules. Transport through gold-BPD-gold and goldFBF-gold junctions have been recently studied in detail using a first-principles self-consistent matrix Green's function theory of electron transport in molecular junctions, $\stackrel{7.8}{=}$ from which we obtain the voltage-dependent background charge $e n_{x}$ induced by the charge transfer from the molecule to the nanoparticle and the transmission coefficient for electron tunneling through the metal-molecule-nanoparticle junction ${ }^{17}$ The charge transfer at zero bias gives the intrinsic background charge $e n_{x 0}$, while its derivative with respect to bias voltage gives the capacitance of the molecular junction 18 The calculated dimensionless conductance, capacitance and the intrinsic background charge for BPD (FBF) molecular junction are 0.0183 (0.0023), $0.037(0.043)$ aF and $-0.623(-0.805)$ number of electrons respectively. Note the total intrinsic background charge on the nanoparticle is the sum of contributions from the two molecular junctions. Despite the shorter length of the FBF molecule, the resistance of the FBF junction is much higher than that of the BPD molecule due to the less favorable energy-level lineup relative to the gold Fermi-level ${ }^{7.8}$

Given the transmission coefficient and capacitance of the molecular junction, the rate equation (6) is solved using

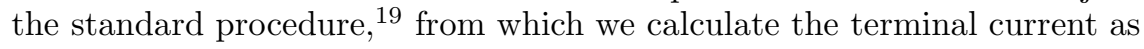

$$
I_{L(R)}=2 \pi e \sum_{n} P_{n}\left[\alpha_{L(R)}^{+}\left(\Delta_{n}\right)-\alpha_{L(R)}^{-}\left(\Delta_{n-1}\right)\right] .
$$

The average excess charge $e\langle\hat{N}\rangle=e \sum_{N} N P_{N}$ gives the net charge transferred onto the nanoparticle due to the discrete electron tunneling across the molecular bridges, while the average charge $e\left(\langle\hat{N}\rangle-n_{x}\right)$ gives the charging configuration which determines the electrostatic energy cost for charging the nanoparticle.

Figs. 2 and 3 show the calculated results for single-electron tunneling through a $10 \mathrm{~nm}$-diameter gold nanoparticle connected to gold electrodes through two BPD or FBF bridges at temperatures of $10(K)$ and $300(K)$ respectively. The self-capacitance of the nanoparticle is evaluated using the electrostatic formula for a conducting sphere in front of a conducting plane, ${ }^{20}$ which gives a charging energy of $E_{C}=88(\mathrm{meV})$. The intrinsic background charge $n_{x 0}$ on the central nanoparticle is closer to half integer in the case of the FBF molecule (-1.61) than that of the BPD molecule $(-1.25)$, so the Coulomb gap in the current/conductance-voltage characteristics is smaller. Note at low temperature the average excess electron $\langle\hat{N}\rangle$ on the nanoparticle at low bias equals the nearest integer to $n_{x 0}$. Since the background charge $n_{x}$ varies slowly with the applied bias voltage within the voltage range studied, both the average charge and the average excess charge show similar voltage dependence. The peaks in the conductance-voltage characteristics correlate with the change in the average excess electron on the nanoparticle at low temperature.

Fig. [4 shows the calculated results for single-electron tunneling through a $10 \mathrm{~nm}$-diameter gold nanoparticle connected to left gold electrode through BPD molecule and connected to right gold electrode through FBF molecule. Since $R_{F B F} / R_{B P D} \gg 1$, varying bias voltage leads to stepwise charging of the central nanoparticle at low temperature. Here the intrinsic background charge $n_{x 0} \approx-1.43$ and $C_{F B F} / C_{B P D}>1$, the shape of the Coulomb staircase 
corresponds to case $\mathbf{I}$ as discussed by Hanna and Tinkham 10 and is well understood. The small Coulomb gap is again due to the fact that $n_{x 0}$ is close to half integer.

To conclude, we have presented a microscopic theory of single-electron tunneling through molecular-assembled metallic nanoparticles, which combines the theory of transport through a molecular junction with the description of the charging dynamics on the nanoparticle. This allows us to take full account of the microscopic description of the electronic processes in the metal-molecule-nanoparticle junction as well as the strong Coulomb interaction on the nanoparticle.

This work was supported by the DARPA Molectronics program, the DoD-DURINT program and the NSF Nanotechnology Initiative.

* Corresponding author. Email: ayxue@chem.nwu.edu

${ }^{1}$ C.P. Collier, T. Vossmeyer and J.R. Heath, Annu. Rev. Phys. Chem. 49, 371 (1998); C.B. Murray, C.R. Kagan and M.G. Bawendi, Annu. Rev. Mater. Sci. 30, 545 (2000).

2 J.J. Storhoff and C.A. Mirkin, Chem. Rev. 99, 1849 (1999).

3 R.P. Andres, T. Bein, M. Dorogi, S. Feng, J.I. Henderson, C.P. Kubiak, W. Mahoney, R.G. Osifchin and R. Reifenberger, Science 272, 1323 (1996).

4 S.H.M. Persson, L. Olofsson and L. Gunnarsson, Appl. Phys. Lett. 74, 2546 (1999); J.R. Petta, D.G. Salinas and D.C. Ralph, ibid. 77, 4419 (2000); T. Ohgi, H.-Y. Sheng, Z.-C. Dong, H. Nejoh and D. Fujita, ibid. 79, 2453 (2001); C.A. Berven, M.N. Wybourne, L. Clarke, L. Longstreth, J.E. Hutchinson and J.L. Mooster, J. Appl. Phys. 92, 4513 (2002); K.-H. Müller, J. Herrmann, B. Raguse, G. Baxter and T. Reda, Phys. Rev. B 66, 75417 (2002).

5 D.V. Averin and K.K. Likharev, in Mesocopic Phenomena in Solids, edited by B.L. Altshuler, P.A. Lee and R.A. Webb (Elsevier, Amsterdam, 1991); G.-L. Ingold and Yu.V. Nazarov, in Single Charge Tunneling, edited by H. Grabert and M.H. Devoret (Plenum, New York, 1992).

${ }^{6}$ C. Joachim, J. K. Gimzewski and A. Aviram, Nature 408, 541 (2000); A. Nitzan and M.A. Ratner, Science 300, 1384 (2003).

7 Y. Xue, S. Datta and M.A. Ratner, J. Chem. Phys. 115, 4292 (2001); Chem. Phys. 281, 151 (2002).

8 Y. Xue and M.A. Ratner, Phys. Rev. B 68, 115406 (2003); to be published.

9 M. Büttiker, J. Phys.: Condens. Matter 5, 9379 (1993).

10 A.E. Hanna and M. Tinkham, Phys. Rev. B 44, 5919 (1991).

11 H. Schoeller and G. Schön, Phys. Rev. B 50, 18436 (1994); Physica 203, 423 (1994).

12 J. König, H. Schoeller and G. Schön, Phys. Rev. Lett. 78, 4482 (1997); Phys. Rev. B 58, 7882 (1998).

13 M.H. Devoret, D. Esteve, H. Grabert, G.-L. Ingold, H. Pothier and C. Urbina, Phys. Rev. Lett. 64, 1824 (1990).

14 Y. Meir and N.S. Wingreen, Phys. Rev. Lett. 68, 2512 (1992); H. Haug and A-P. Jauho, Quantum Kinetics in Transport and Optics of Semiconductors (Springer-Verlag, Berlin, 1996).

15 Y. Xue and M.A. Ratner, Materials Research Society Proceedings 735, C5.5 (2003).

16 Depending on the molecules chosen, the molecular junction may become sufficiently conducting such that higher order effects including quantum fluctuation of charges on the nanoparticle and cotunneling effects become important. The microscopic theory presented here provides a systematic way of investigating such effects following the procedures described in Refs. 11 and 12. But for single-electron device applications, molecular junction with high resistance is desirable and the lowest-order perturbation theory is sufficient. The choice of the molecules studied here reflects this consideration.

17 Since the metal-molecule interaction is a local phenomenon, we neglect the curvature of the nanoparticle surface and calculate the electronic structure of metal-molecule-nanoparticle junction in the same way as that of the metal-molecule-metal junction in Refs. 7 and 8 .

18 The self-consistent calculation is performed in a "extended molecule" region including both the molecule and the perturbed surface atoms of the electrodes and the nanoparticle. The transferred charge and therefore the background charge $n_{x}$ is obtained by integrating the (self-consistent) electron density distribution over the region occupied by the perturbed surface atoms of the nanoparticle.

19 M. Amman, R. Wilkins, E. Ben-Jacob, P.D. Maker and R.C. Jaklevic, Phys. Rev. B 43, 1146 (1991).

20 C. Wasshuber, Computational Single-Electronics (Springer-Verlag, Wien, 2001). 


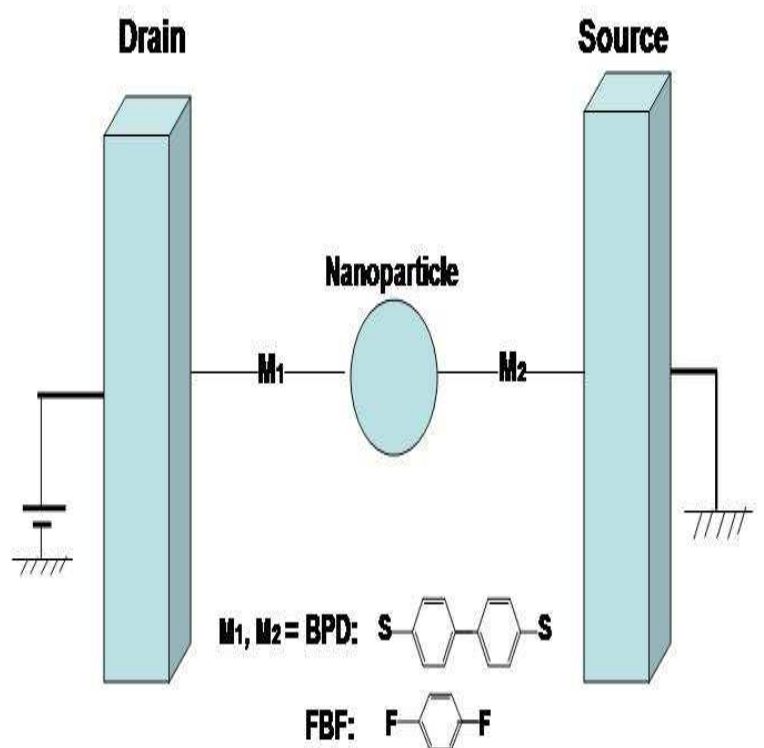

FIG. 1: Schematic illustration of a metallic nanoparticle connected to the source and drain electrodes through two molecular bridges.

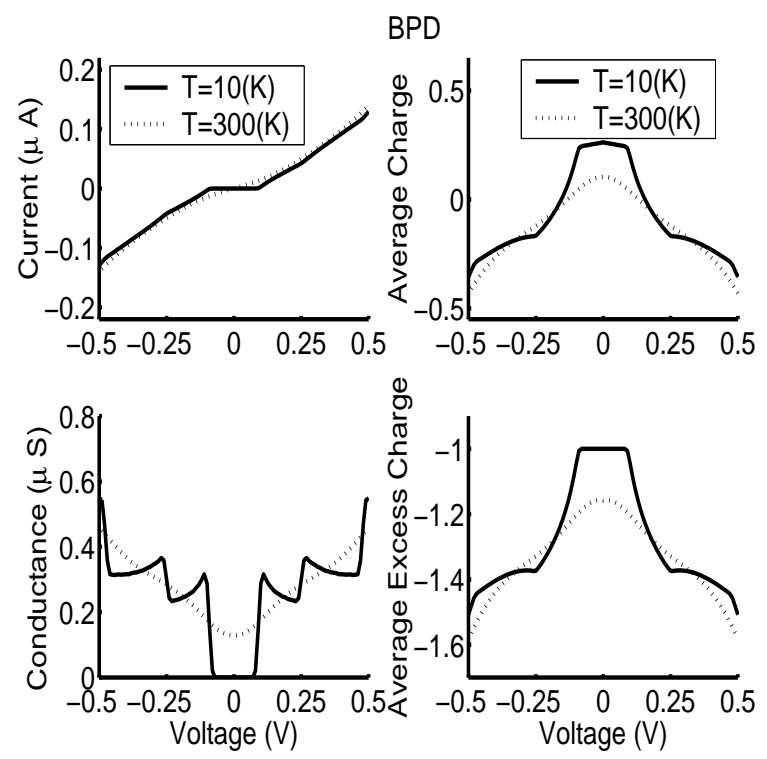

FIG. 2: Single-electron tunneling through a $10 \mathrm{~nm}$-diameter gold nanoparticle connected to the source and drain electrodes through the BPD molecule. Left figure shows the current-voltage and conductance-voltage characteristics. Right figure shows the average charge $e\left(\hat{N}-n_{x}\right)$ and average excess charge $e \hat{N}$ on the nanoparticle in unit of electron charge $e$. 


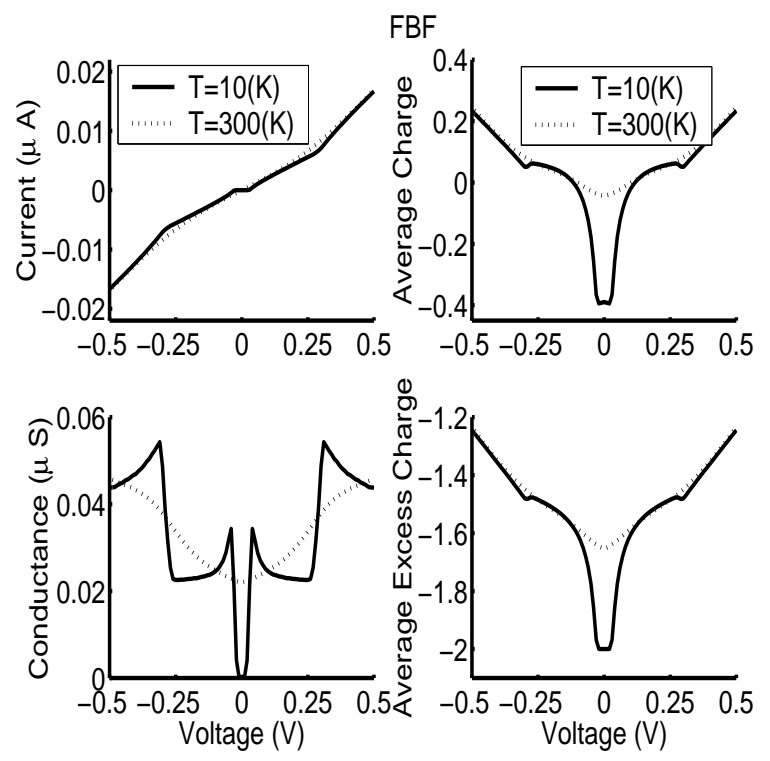

FIG. 3: Single-electron tunneling through a $10 \mathrm{~nm}$-diameter gold nanoparticle connected to the source and drain electrodes through the FBF molecule as in Fig. [2]

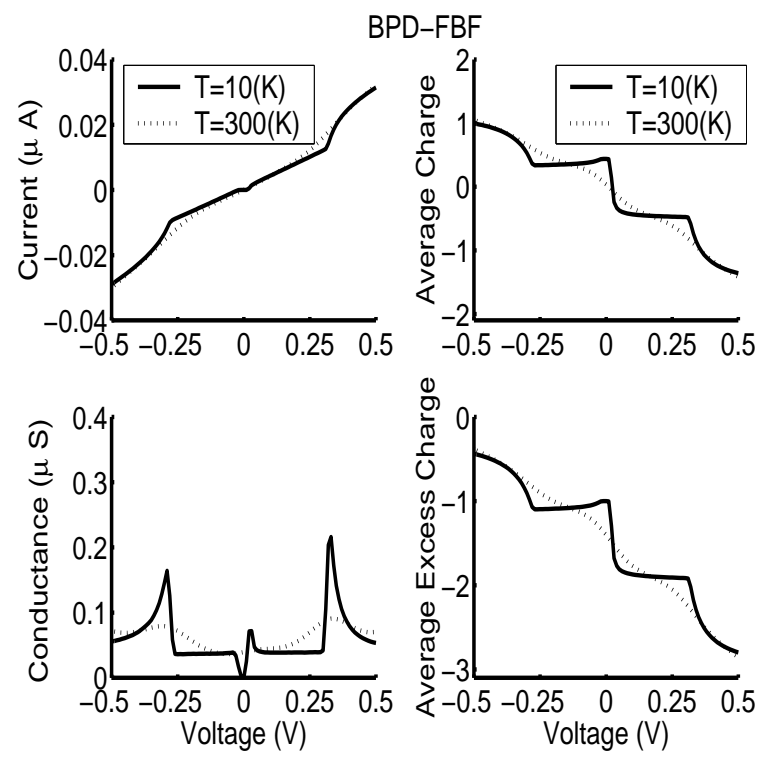

FIG. 4: Single-electron tunneling through a $10 \mathrm{~nm}$-diameter gold nanopartcile connected to the source electrode through the BPD molecule and the drain electrode through the FBF molecule as in Fig. 2 